\title{
Incremental shuttle walk test in the assessment of patients for heart transplantation
}

\author{
M E Lewis, C Newall, J N Townend, S L Hill, R S Bonser
}

\begin{abstract}
Objective-To compare the incremental shuttle walk test (ISWT) with treadmill exercise testing (TT) derived measurement of peak oxygen consumption (peak $\mathrm{VO}_{2}$ ) in patients undergoing assessment for cardiac transplantation.

Design-Prospective comparison. All investigations occurred during a single period of admission for transplant assessment.

Setting-Single UK cardiothoracic transplantation unit.

Patients-25 patients recruited (21 men). Mean age was 53 years.

Interventions-Patients underwent two TT of peak $\mathrm{VO}_{2}$ using the modified Naughton protocol and three (one practice) ISWT. Investigations were performed on consecutive days.

Main outcome measures-Main outcome measures were repeatability of TT and ISWT assessments; relation between peak $\mathrm{VO}_{2}$ and distance walked in the ISWT; and receiver operating characteristic (ROC) analysis to establish a distance walked in the ISWT that predicted which patients would have a peak $\mathrm{VO}_{2}$ greater than $14 \mathrm{ml} / \mathrm{min} / \mathrm{kg}$.

Results-Both the ISWT and the TT were highly reproducible. Following the first practice walk, mean (SD) ISWT distances were 400.0 (146) $\mathrm{m}$ (ISWT2) and 401.3 (129) $\mathrm{m}$ (ISWT3), $r=0.90, \mathrm{p}<0.0001$. Mean peak $\mathrm{VO}_{2}$ by TT was $15.2(4.4) \mathrm{ml} / \mathrm{kg} / \mathrm{min}(\mathrm{TT} 1)$ and $15.0(4.4) \mathrm{ml} /$ $\mathrm{kg} / \mathrm{min}$ (TT2), $r=0.83, \mathrm{p}<0.0001$. The results revealed a strong correlation between distance covered in the ISWT and peak $\mathrm{VO}_{2}$ obtained during TT $(r=0.73, \mathrm{p}=0.0001)$. ROC analysis showed that a distance walked of $450 \mathrm{~m}$ allowed the selection of patients with a peak $\mathrm{VO}_{2}$ of over $14 \mathrm{ml} / \mathrm{min} / \mathrm{kg}$.

Conclusions-This work confirms the utility of the ISWT in the assessment of exercise capacity in patients with severe heart failure undergoing assessment for cardiac transplantation. ISWT may provide a widely applicable surrogate measure for peak $\mathrm{VO}_{2}$ estimation in this population. Shuttle distance walked may therefore allow the convenient, serial assessment of patients with heart failure before referral for transplantation.

(Heart 2001;86:183-187)
\end{abstract}

Keywords: incremental shuttle walk; peak oxygen consumption; cardiac transplantation

Cardiac transplantation is an effective treatment for severe heart failure improving both quality and quantity of life. ${ }^{1}$ Unfortunately, donor organ scarcity means that only a small fraction of patients with heart failure can be offered transplantation. The selection of appropriate patients is difficult because many of the features of heart failure such as left ventricular function are only weakly related to symptoms and are poor prognostic discriminators. Of the available methods for assessing prognosis in heart failure, the assessment of exercise capacity by measurement of maximal oxygen consumption (peak $\mathrm{VO}_{2}$ ) during treadmill or cycle ergometer exercise testing is perhaps the most useful and widely used. It provides an accurate and reproducible measure of both symptomatic status and prognosis in patients with heart failure $^{23}$ and when combined with other outcome predictors allows accurate outcome estimation. ${ }^{4}$ Unfortunately, the equipment is expensive and not widely available outside large institutions. In addition, the test is limited in its application, firstly by the need for an incremental exercise protocol, which is an abnormal exercise that many patients find difficult; secondly, by the required facemask or mouthpiece, which may be poorly tolerated; and finally, by the need for staff skilled in both exercise physiology and in patient motivation techniques to obtain meaningful results.

Thus, there is a clear requirement for an alternative inexpensive and simple measure of exercise capacity that is reproducible and prognostically reliable.

The incremental shuttle walk test (ISWT) was originally developed to assess patients with chronic obstructive pulmonary disease and requires patients to walk at a gradually increasing speed until they reach a symptom limited maximum. ${ }^{5}$ Furthermore, a clear relation has been shown in patients with chronic obstructive pulmonary disease between distance walked in the ISWT and peak $\mathrm{VO}_{2} .{ }^{6}$ The wide range of walking speed in the ISWT allows the accommodation of all ambulant patients, from those with minimal disability to those with more severe symptoms.

We report a prospective comparison of the ISWT with the measurement of $\mathrm{Vo}_{2}$ peak during treadmill exercise testing (TT) in 25 patients undergoing assessment for cardiac transplantation at our institution. We first sought to establish the reproducibility of each test and subsequently examined their relation. 
Table 1 Patient characteristics for the entire group and for the two groups established by dividing the patient population into those with (group 2) and those without (group 1) peak oxygen consumption $>14 \mathrm{ml} / \mathrm{min} / \mathrm{kg}$

\begin{tabular}{lllll}
\hline & $\begin{array}{l}\text { Total group } \\
(n=25)\end{array}$ & Group 1 $(n=8)$ & Group 2 $(n=17)$ & p Value \\
\hline Male (\%) & 84 & 87.5 & 82.4 & 0.67 \\
Age (years) & $53(8)$ & $51(12)$ & $54(6)$ & 0.35 \\
LVEF (\%) & $27.8(12.2)$ & $26.3(18.3)$ & $28.5(8.7)$ & 0.67 \\
LVEDD (cm) & $6.6(1.2)$ & $6.9(1.0)$ & $6.4(1.3)$ & 0.5 \\
Cardiac index $\left(1 / \mathrm{min}^{2} \mathrm{~m}^{2}\right)$ & $2.2(0.5)$ & $1.8(0.36)$ & $2.4(0.39)$ & 0.03 \\
Serum sodium (mmol/1) & $138(3.7)$ & $137.8(3.5)$ & $137.8(3.9)$ & 0.98 \\
NYHA & II-III & III-IV & II-III & 0.0005 \\
PCWP (mm Hg) & $17.2(10.5)$ & $22.2(8.7)$ & $14.6(10.6)$ & 0.11 \\
TPG $(\mathrm{mm} \mathrm{Hg})$ & $7.3(2.2)$ & $7.2(1.06)$ & $7.4(2.6)$ & 0.78 \\
Shuttle distance $(\mathrm{m})$ & $401(147)$ & $287(127)$ & $454(124)$ & 0.0005 \\
Vo $(\mathrm{ml} / \mathrm{min} / \mathrm{kg})$ & $15.2(4.4)$ & $10.0(2.0)$ & $17.6(2.7)$ & - \\
\hline
\end{tabular}

Data are mean (SD). p Values for comparison group 1 versus group 2.

LVEDD, left ventricular end diastolic dimension; LVEF, left ventricular ejection fraction; NYHA, New York Heart Association functional class; PCWP, pulmonary capillary wedge pressure; TPG, transpulmonary gradient; $\mathrm{VO}_{2}$, peak oxygen consumption.

\section{Patients and methods \\ PATIENTS}

Patients undergoing assessment for cardiac transplantation were recruited between January and September 1999. All ambulatory patients were invited to take part but patients requiring inotropic support were excluded. All patients underwent an initial treadmill assessment for measurement of peak $\mathrm{VO}_{2}$ using a modified Naughton protocol as part of the routine assessment process. Patients unable to complete a single two minute stage (11 patients) were excluded. Twenty five (21 men) of 48 eligible patients assessed during this period consented to take part in this study. The study was approved by the local research ethics committee and written consent was obtained from all patients. The mean age was 53 years (range 33-69 years). Fifteen of the 25 patients had a diagnosis of ischaemic cardiomyopathy and 10 had a dilated non-ischaemic cardiomyopathy.

\section{STUDY DESIGN}

All investigations occurred during a single period of admission for transplant assessment according to the following schedule: day 1,9 am TT (TT1), 4 pm ISWT twice (one practice (ISWT1), one formal assessment (ISWT2)); day 2, 2 pm ISWT once (ISWT3); day 3, 11 am TT (TT2). The timings of tests had to be adjusted, on occasion, to accommodate other transplantation assessment investigations. All medications were continued as routine. $\mathrm{Pa}-$ tients who used sublingual nitrates were given one dose five minutes before each test.

\section{SHUTTLE WALKING TEST}

The patients performed three ISWT. ${ }^{5}$ The design of the test was as follows: the patients were required to walk up and down a $10 \mathrm{~m}$ course at a speed dictated by signals from an audio tape recorder. The walking speed was increased by a small increment at 1 minute intervals. The test was terminated either by the patient if he or she felt too breathless to continue at the desired speed or by the operators if the patient failed to complete a $10 \mathrm{~m}$ length (shuttle) in the time allowed. During the test, heart rate was recorded with a Polar heart rate monitor.
TREADMILL PROTOCOL

Patients were subjected to an incremental TT using the modified Naughton protocol, which increases in two minute stages. Exercise was discontinued when the patient was unable to maintain the imposed workload (manifest by the inability to maintain speed consistently on the treadmill, or by development of significant exercise induced chest pain or ECG disturbance). Patients breathed through a two way non-rebreathable valve supported by headgear supplied for use with the treadmill. Inspired ventilation was monitored continuously by a Fleish pneumatograph positioned at the inspiratory port of the two way mouthpiece valve. Expired gas was passed through a mixing chamber, and fractional $\mathrm{O}_{2}$ and $\mathrm{CO}_{2}$ were recorded continuously by rapid response analysers (Zirconia cell analyser and Infra red rapid response analyser, for $\mathrm{O}_{2}$ and $\mathrm{CO}_{2}$ respectively, Morgan Medical Ltd, Kent, UK). Peak $\mathrm{VO}_{2}$ and $\mathrm{CO}_{2}$ output were calculated at 30 second intervals throughout the test. Peak $\mathrm{Vo}_{2}$ was defined as the maximal rate of $\mathrm{O}_{2}$ consumption in $\mathrm{ml} / \mathrm{min} / \mathrm{kg}$. Anaerobic threshold was defined to have occurred when there was a systematic increase in the ventilatory equivalent of $\mathrm{O}_{2}$ with a corresponding increase in $\mathrm{CO}_{2}$ output. Patients stopped because of shortness of breath (50\%), limb fatigue (20\%), and chest pain $(30 \%)$. Reasons for stopping the ISWT and TT were identical for each patient for each test.

\section{PATIENT PREFERENCE}

Patient preference was determined by the responses to a questionnaire administered by a technician.

STATISTICAL ANALYSIS

All statistical analysis was performed on a standard personal computer running the ARCUS software package (Addison Wesley Longman, Cambridge, UK). Continuous variables were assessed for normal distribution and compared by the two way unpaired $t$ test. Receiver operating characteristic (ROC) analysis was performed using the methodology of Altman. ${ }^{7}$ Regression and correlation analysis was performed as described by Bland and Altman. ${ }^{8}$ Values are presented as mean (SD) unless otherwise stated. Significance was assumed when $\mathrm{p}<0.05$.

\section{Results}

Clinical and haemodynamic variables for the patients are shown in table 1. During TT only three of the 25 patients achieved anaerobic threshold, despite firm encouragement. All of these patients reached anaerobic threshold on both $\mathrm{TT} \mathrm{VO}_{2}$ estimations. The mean respiratory exchange ratio for all patients was 0.95 (0.12).

\section{REPRODUCIBILITY}

Both the ISWT and the TT were highly reproducible (fig 1 and 2). Following the first practice walk (347 (140) m), mean ISWT distances were 400.0 (146) $\mathrm{m}$ (ISWT2) and 401.3 


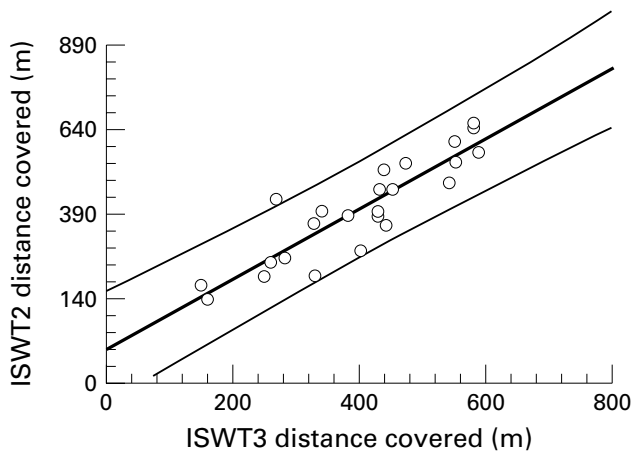

Figure 1 Distance walked by each patient $(m)$ in incremental shuttle walk test 2 (ISWT2) v test 3 (ISWT3) $(r=0.90, p<0.0001)$. The $95 \%$ prediction interval is shown.

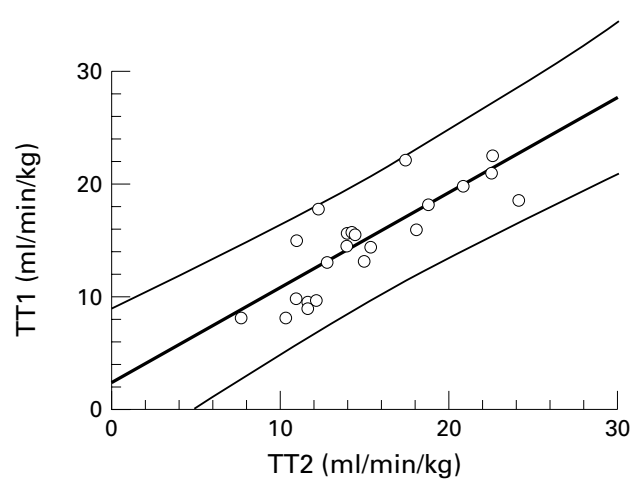

Figure 2 Peak oxygen consumption $(\mathrm{ml} / \mathrm{min} / \mathrm{kg}$ ) achieved in treadmill test 1(TT1) v treadmill test 2 (TT2) $(r=$ $0.83, p<0.0001)$. The $95 \%$ prediction interval is shown.

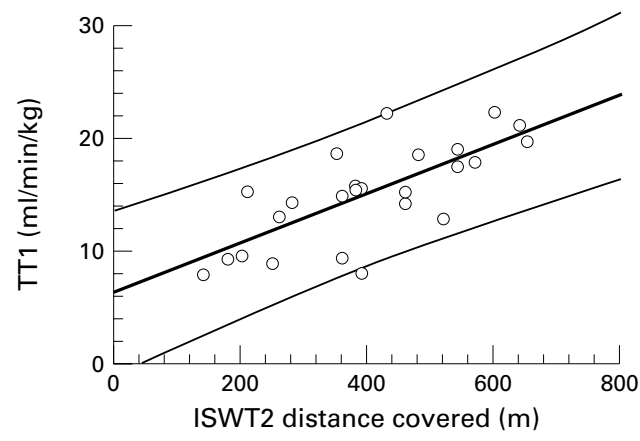

Figure 3 Distance walked in incremental shuttle walk test 2 (ISWT2) ( $\mathrm{m})$ v peak oxygen consumption $(\mathrm{ml} / \mathrm{min} / \mathrm{kg})$ achieved in treadmill test 1 (TT1) $(r=0.73, p=0.0001)$. The $95 \%$ prediction interval is shown.

(129) $\mathrm{m}$ (ISWT3), $r=0.90, \mathrm{p}<0.0001$. Mean $\mathrm{VO}_{2}$ by TT was $15.2(4.4) \mathrm{ml} / \mathrm{kg} / \mathrm{min}$ (TT1) and $15.0(4.4) \mathrm{ml} / \mathrm{kg} / \mathrm{min}$ (TT2), $\mathrm{r}=0.83$, $\mathrm{p}<0.0001$.

RELATION BETWEEN PEAK $\mathrm{VO}_{2}$ AND ISWT DISTANCE

The results revealed a strong correlation between distance covered in the ISWT and peak $\mathrm{VO}_{2}$ obtained during the TT (fig 3). This is represented by the regression equation $\mathrm{Vo}_{2}=$ $0.022 \times$ Dist +6.4 , where $\mathrm{Vo}_{2}$ is peak $\mathrm{Vo}_{2}(\mathrm{ml} /$ $\mathrm{min} / \mathrm{kg}$ ) and Dist is distance walked during the ISWT $(\mathrm{m})(r=0.73, \mathrm{p}=0.0001)$. Mean peak heart rates for both tests were similar $\left(\mathrm{VO}_{2}, 128\right.$ (25) beats/min; ISWT, 129 (23) beats/min, $r=0.68, \mathrm{p}=0.0004)$.

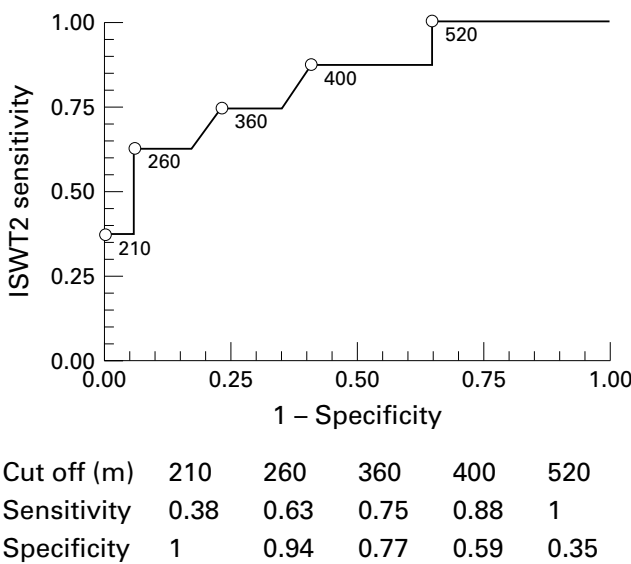

Figure 4 Receiver operating characteristic plot for peak oxygen consumption $>14 \mathrm{ml} / \mathrm{min} / \mathrm{kg}$ comparing incremental shuttle walk test 2 (ISWT2) with treadmill test 1 (TT1).

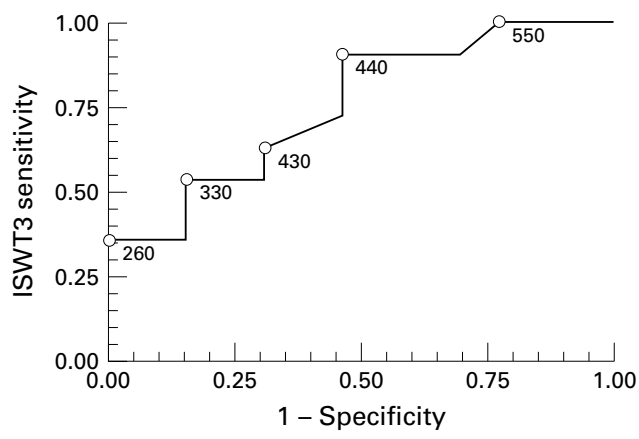

$\begin{array}{llllll}\text { Cut off }(\mathrm{m}) & 260 & 330 & 430 & 440 & 550 \\ \text { Sensitivity } & 0.23 & 0.54 & 0.69 & 0.85 & 1.0 \\ \text { Specificity } & 1.0 & 0.9 & 0.64 & 0.55 & 0.36\end{array}$

Figure 5 Receiver operating characteristic plot for peak oxygen consumption $>14 \mathrm{ml} / \mathrm{min} / \mathrm{kg}$ comparing incremental shuttle walk test 3 (ISWT3) with treadmill test 2 (TT2).

Of the other variables routinely measured at cardiac transplantation assessment (table 1), only resting cardiac index correlated with peak $\mathrm{VO}_{2}$ and shuttle distance $(r=0.58, \mathrm{p}=0.005$ and $r=0.43, \mathrm{p}=0.05$, respectively) on simple linear regression. Backwards stepwise multiple linear regression, of the variables listed in table 1 , identified only distance covered in the ISWT as a predictor of peak $\mathrm{Vo}_{2}(\mathrm{p}=0.04)$.

DISCRIMINATING POWER

To assess the ability of the ISWT to discriminate between patients with a peak $\mathrm{VO}_{2}$ of less than or greater than $14 \mathrm{ml} / \mathrm{min} / \mathrm{kg}$, ROC curves were constructed (fig 4, ISWT2 and TT1, and fig 5, ISWT3 and TT2). The data are presented separately to avoid bias resulting from the correlation of data sets. The two curves show that the ISWT threshold providing optimal sensitivity and specificity is not precisely determined by a single comparison. From the ROC curves, it appears that an ISWT threshold of between 370 and $430 \mathrm{~m}$ provides sensitivities and specificities of approximately $75 \%$ for predicting which patients have a mean peak $\mathrm{VO}_{2}$ of over $14 \mathrm{ml} / \mathrm{min} / \mathrm{kg}$. 
ISWT AND PROGNOSTIC INDICES OF HEART

FAILURE

The mean peak $\mathrm{VO}_{2}$ was 15.2 (4.4) $\mathrm{ml} / \mathrm{min} / \mathrm{kg}$. On the basis of previous work, ${ }^{2}$ we divided the patients into two groups: group 1 , with a $\mathrm{VO}_{2}$ of $\leqslant 14 \mathrm{ml} / \mathrm{min} / \mathrm{kg}$; and group 2 , with a $\mathrm{Vo}_{2}$ of $>14 \mathrm{ml} / \mathrm{min} / \mathrm{kg}$. Of all variables measured, New York Heart Association (NYHA) functional class and resting cardiac index were statistically different between groups. In addition, the distance covered in the ISWT was highly significantly different between group 1 and group 2 .

Both pulmonary capillary wedge pressure ( $\mathrm{p}=0.03$ ) and NYHA functional class ( $p=0.02$ ) were significantly different between patients who could walk further than $450 \mathrm{~m}$ and those who managed $450 \mathrm{~m}$ or less.

\section{PATIENT PREFERENCE}

Twenty four of the 25 patients stated that they preferred the shuttle walk test.

\section{Discussion}

This study has shown that the ISWT appears to provide a reliable and reproducible measure of exercise tolerance in severe heart failure. The close correlation of ISWT distance with peak $\mathrm{VO}_{2}$ indicates that the results may be useful as a prognostic index in patients with heart failure. ROC analysis shows that an ISWT distance of $450 \mathrm{~m}$ is a good discriminator of patients with a $\mathrm{VO}_{2}$ of more than $14 \mathrm{ml} / \mathrm{min} /$ $\mathrm{kg}$. ${ }^{9}$ This peak $\mathrm{VO}_{2}$ is widely considered to be an important indicator of prognosis in assessment for cardiac transplantation; patients with values below this level have a high short term mortality. ${ }^{2} 310$

Advantages of the ISWT over the conventional TT $\mathrm{VO}_{2}$ assessment include better patient tolerance, ease of administration, and no requirement for expensive equipment. However, as with all tests of exercise tolerance, it is subject to variations in patient motivation and the investigators' ability to ensure that patients reach their symptomatic limits. The test appears to have wide applicability. Recent work has shown that the ISWT can be used as a reproducible measure of exercise capability $^{511}$ and is comparable with peak $\mathrm{VO}_{2}$ in patients with lung disease. ${ }^{6}$ Similar results have been reported in patients with cardiac pacemakers. ${ }^{12}$

Two previous studies have compared the ISWT with peak $\mathrm{VO}_{2}$ exercise testing in patients with heart failure. In patients undergoing assessment at a heart failure clinic, $\mathrm{VO}_{2} \mathrm{max}$ and ISWT distance covered showed a good correlation $(\mathrm{r}=0.84, \mathrm{p}<0.0001)$ in a population of 50 patients with mild to moderate heart failure (mean $\mathrm{VO}_{2} 17.9$ (6.1) ml/kg/ min). ${ }^{13}$ In a study comparing cycle ergometer derived peak $\mathrm{VO}_{2}$ with the six minute walk test and the ISWT, Morales and colleagues ${ }^{14}$ also showed a close $(r=0.83)$ correlation between $\mathrm{VO}_{2}$ and ISWT distance covered in patients with moderate heart failure (mean $\mathrm{VO}_{2} 17.8$ (4.4) $\mathrm{ml} / \mathrm{kg} / \mathrm{min}$ ). As in our study they found that the ISWT was reproducible after just one walk. However, the mean peak $\mathrm{Vo}_{2}$ in both of these studies was significantly higher than that of our patients, suggesting less severe heart failure. In addition, the use of a cycle ergometer rather than a treadmill may underestimate peak $\mathrm{VO}_{2} .{ }^{15}$ Although the patients studied by Morales and colleagues ${ }^{14}$ had less severe heart failure than our study group, a cut off distance of $450 \mathrm{~m}$ also defined the patients with a $\mathrm{VO}_{2}$ of $>14 \mathrm{ml} / \mathrm{kg} / \mathrm{min}$. Again, the ISWT result was the only predictor of peak $\mathrm{VO}_{2}$ by multiple regression.

Our work confirms the utility of the ISWT in the assessment of exercise capacity in patients with severe heart failure undergoing assessment for cardiac transplantation. In addition ISWT may provide a widely applicable surrogate for peak $\mathrm{VO}_{2}$ estimation in this population. The shuttle walk test gives an incremental workload provoking maximal performance in the same way as a conventional TT. Further large scale studies are required, but if our results are confirmed, shuttle distance walked may allow the convenient, serial assessment of patients with heart failure before referral for transplantation.

\section{STUDY LIMITATIONS}

Unfortunately only a small number of patients reached an anaerobic threshold during formal TT. Patients with severe heart failure often have oscillatory or sustained hyperventilation. This results in excess $\mathrm{CO}_{2}$ elimination from the onset of exercise. ${ }^{16}$ This makes the non-invasive determination of anaerobic threshold difficult in these patients. In addition, a number of our patients with ischaemic cardiomyopathy terminated their exercise because of angina. ${ }^{6}$ Despite this limitation, the exercise workload in the TT and ISWT appear to be comparable because maximal heart rates were similar $(75 \%$ and $77 \%$ predicted maximum, respectively). Moreover, recent work has shown that the prognostic power of a low peak $\mathrm{VO}_{2}$ in patients with severe heart failure is maintained even when anaerobic threshold is not reached. ${ }^{17}$

The help and assistance of Miss S. Beer in the recruitment of patients for this study is gratefully recognised.

1 UKTSSA. Cardiothoracic organ transplant audit 19851995. http://www.uktransplant.org.uk

2 Mancini DM, Eisen H, Kussmaul W, et al. Value of peak exercise oxygen consumption for optimal timing of cardiac transplantation in ambulatory patients with heart failure. Circulation 1991; 83:778-86.

3 Mancini D, LeJemtel T, Aaronson K. Peak VO(2): a simple yet enduring standard Circulation 2000; 101:1080-2.

4 Aaronson KD, Schwartz JS, Chen TM, et al. Development Aaronson KD, Schwartz JS, Chen TM, et al. Development
and prospective validation of a clinical index to predict survival in ambulatory patients referred for cardiac transplant evaluation Circulation 1997; 95:2660-7.

5 Singh SJ, Morgan MD, Scott S, et al. Development of a shuttle walking test of disability in patients with chronic airways obstruction. Thorax 1992;47:1019-24.

6 Singh SJ, Morgan MD, Hardman AE, et al. Comparison of oxygen uptake during a conventional treadmill test and the shuttle walking test in chronic airflow limitation. Eur Respir f 1994; 7:2016-20.

7 Altman D. Practical statistics for medical research. New York: Chapman and Hall 1991.

8 Bland JM, Altman DG. Statistical methods for assessing agreement between two methods of clinical measurement. Lancet 1986;i:307-10.

9 Swets JA. Measuring the accuracy of diagnostic systems. Science 1988;240:1285-93. 
10 Myers J, Gullestad L, Vagelos R, et al. Clinical, hemodynamic, and cardiopulmonary exercise test determinants of survival in patients referred for evaluation of heart failure Ann Intern Med 1998;129:286-93.

11 Singh S. The use of field walking tests for assessment of functional capacity in patients with chronic airways obstruction. Physiotherapy 1992;78:102-4.

12 Payne GE, Skehan JD. Shuttle walking test: a new approach for evaluating patients with pacemakers. Heart 1996;75 $414-8$

13 Keell SD, Chambers JS, Francis DP, et al. Shuttle-walk test to assess chronic heart failure. Lancet 1998;352:705.
14 Morales FJ, Martinez A, Mendez M, et al. A shuttle walk test for assessment of functional capacity in chronic heart test for assessment of functional cap
failure. Am Heart $\mathcal{F}$ 1999;138:291-8.

15 Myers J, Buchanan N, Walsh D, et al. Comparison of the ramp versus standard exercise protocols. $\mathrm{f}$ Am Coll Cardiol 1991;17:1334-42.

16 Francis GS, Kraemer MD. Exercise testing for patients with heart failure. ACC Curr $\mathcal{F}$ Rev 1995;4:59-63.

17 Opasich C, Pinna GD, Bobbio M, et al. Peak exercise oxygen consumption in chronic heart failure: toward efficient use in the individual patient. $f \mathrm{Am}$ Coll Cardiol 1998;31:766-75.

\section{IMAGES IN CARDIOLOGY}

\section{Coronary artery rupture treated with microcoil occlusion}

Coronary artery rupture is a rare complication of balloon angioplasty. Techniques used in its management include emergency surgery, covered stent deployment, and microcoil vessel occlusion. The latter has been reported twice. We report a further case of successful coil occlusion of a ruptured coronary artery following angioplasty.

A 53 year old man presented with unstable angina. Angiography demonstrated occlusion of the first obtuse marginal artery. Balloon angioplasty using a $2 \mathrm{~mm}$ balloon was undertaken with deployment of a $2.5 \times 18 \mathrm{~mm}$ stent (S670, Medtronic).

Shortly after stent deployment, the patient became haemodynamically compromised and angiography showed perforation of the obtuse marginal artery distal to the stent site (below left).

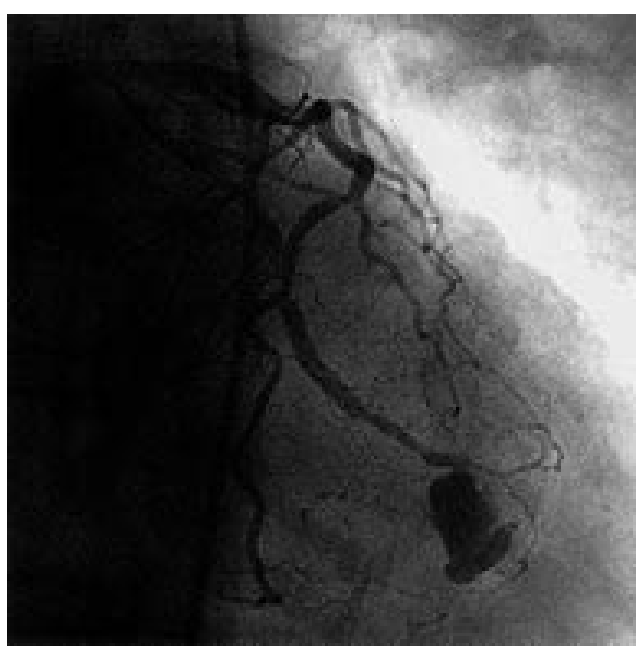

The pericardium was drained percutaneously. The patient continued to haemorrhage at a rate of $50 \mathrm{ml} / \mathrm{min}$ into the pericardium. Covered stent deployment was not considered feasible because of the calibre of the distal vessel at the site of perforation. Coil occlusion of the obtuse marginal artery was therefore performed, using six $2 \times 10 \mathrm{~mm}$ and two $2 \times 5 \mathrm{~mm}$ fibred platinum coils (Target Therapeutics) (below right). There was no further extravasation on contrast injection and no further blood was aspirated from the pericardium. The patient sustained a rise in creatinine kinase to a peak of $444 \mathrm{IU} / 1$, but otherwise made an excellent recovery and was discharged one week later.

$$
\begin{array}{r}
\text { S BANERJEE } \\
\text { R EGDELL } \\
\text { A WATKINSON } \\
\text { R GREENBAUM } \\
\text { susanabanerjee@hotmail.com }
\end{array}
$$

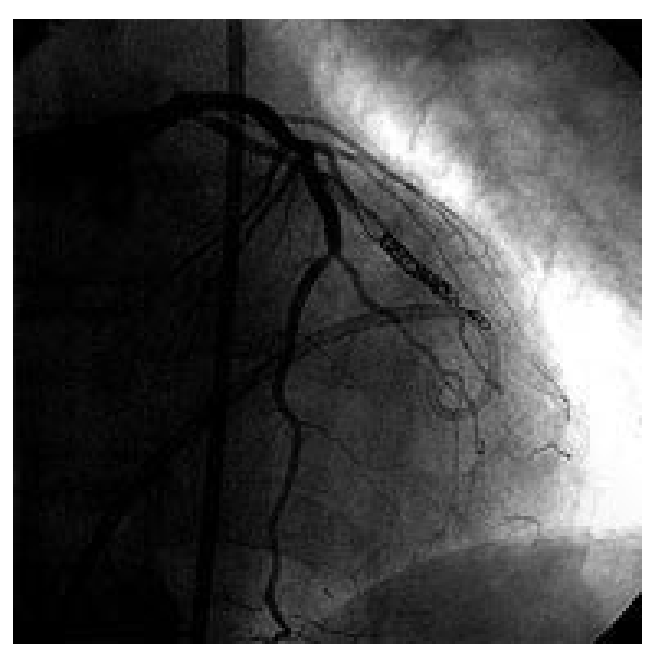

